\title{
Generalized Parton Distributions from Hadronic Observables
}

\author{
S. Ahmad ${ }^{1}$, H. Honkanen ${ }^{1}$, S. Liuti ${ }^{1}$ and S.K. Taneja ${ }^{2}$ \\ 1- University of Virginia - Physics Department \\ 382, McCormick Rd., Charlottesville, Virginia 22904 - USA \\ 2- Ecole Polytechnique, \\ CPHT, F91128 Palaiseau Cedex, France
}

\begin{abstract}
We propose a physically motivated parametrization for the unpolarized generalized parton distributions, $H$ and $E$, valid at both zero and non-zero values of the skewness variable, $\zeta$. At $\zeta=0, H$ and $E$ are determined using constraints from simultaneous fits of experimental data on both the nucleon elastic form factors and the deep inelastic structure functions. Lattice calculations of the higher moments constrain the parametrization at $\zeta>0$. Our method provides a step towards a model independent extraction of generalized distributions from the data that is alternative to the mathematical ansatz of double distributions.
\end{abstract}

\section{Introduction}

Generalized Parton Distributions (GPDs) parametrize the soft contributions in a variety of hard exclusive processes, from Deeply Virtual Compton Scattering (DVCS) to hard exclusive meson production (see $[2,3]$ for reviews). The conceptual idea behind their definition allows one to address a vast, previously inaccessible phenomenology, from the simultaneous description of hadronic structure in terms of transverse spatial and longitudinal momentum degrees of freedom [4], to the the access to the description of angular momentum of partons in nucleons and nuclei via Ji's sum rule [5].

At present, a central issue is the definition of a quantitative, reliable approach beyond the construction of GPDs from specific models and/or particular limiting cases, that can incorporate new incoming experimental data in a variety of ranges of the scale $Q^{2}$, and the four-momentum transfer between the incoming and outgoing protons, $\Delta \equiv(t, \zeta)$. The matching between measured quantities and Perturbative QCD (PQCD) based predictions for DVCS should proceed, owing to specific factorization theorems, similarly to the extraction of Parton Distribution Functions (PDFs) from deep inelastic scattering. A few important caveats are however present since GPDs describe amplitudes and are therefore more elusive observables in experimental measurements. Experiments delivering sufficiently accurate data have, in fact, just begun [6]. The comparison with experiment and the formulation of parametrizations necessarily encompasses, therefore, other strategies using additional constraints, other than from a direct comparison with the data.

We propose a strategy using a combination of experimental data on nucleon form factors, PDFs, and lattice calculations of Mellin moments with $n \geq 1$. The latter, parametrized in terms of Generalized Form Factors (GFFs), were calculated by both the QCDSF [7] and LHPC [8] collaborations for both the unpolarized and polarized cases up to $n=3$, therefore allowing to access the skewness dependence of GPDs. 


\section{Generalized Parton Distributions from Lattice Moments}

GPDs can be extracted most cleanly from DVCS [5]. In this contribution we concentrate on the unpolarized scattering GPDs, $H$, and $E$, from the vector $\left(\gamma_{\mu}\right)$ and tensor $\left(\sigma_{\mu \nu}\right)$ interactions, respectively. We adopt the following set of kinematical variables: $(\zeta, X, t)$, where $\zeta=Q^{2} / 2(P q)$ is the longitudinal momentum transfer between the initial and final protons $\left(\zeta \approx x_{B j}\right.$ in the asymptotic limit, with Bjorken $x_{B j}=$ $\left.Q^{2} / 2 M \nu\right), X=(k q) /(P q)$ is the momentum fraction relative to the initial proton carried by the struck parton, $t=-\Delta^{2}$, is the four-momentum transfer squared. $X$ is not directly observable, it appears in the amplitude as an integration variable $[2,3]$. The need to deal with a more complicated phase space, in addition to the fact that DVCS interferes coherently with the BetheHeitler $(\mathrm{BH})$ process, are in essence the reasons why it is more challenging to extract GPDs from experiment, wherefore guidance from phenomenologically motivated parametrizations becomes important.

We first present a parametrization of $H$ and

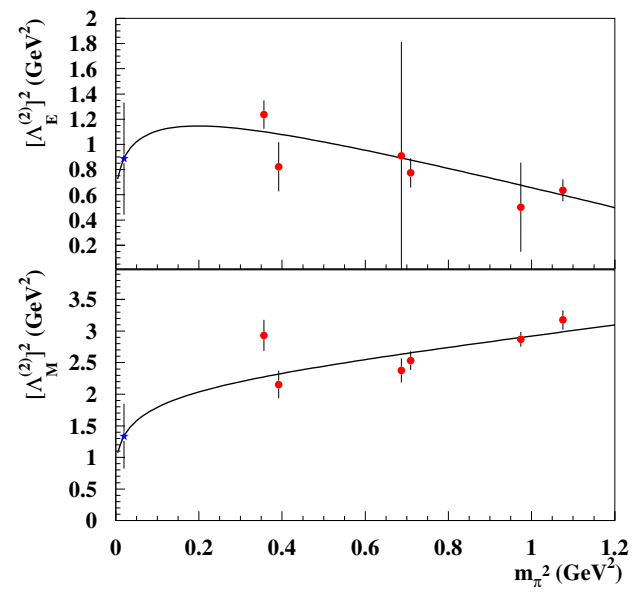

Figure 1: (color online) The dipole masses squared for $n=2$, for the isovector magnetic (lower panel) and electric (upper panel) contributions obtained by performing fits to the lattice results of [7]. The value at the physical pion mass obtained from our fit is also shown (star). $E$ in the flavor Non Singlet (NS) sector, valid in the $X>\zeta$ region, obtained by extending our previous zero skewness form [9], through proper kinematical shifts:

$$
H(X, \zeta, t)=G_{M_{X}}^{\lambda}(X, \zeta, t) R(X, \zeta, t)
$$

(a similar form was used for $E(X, \zeta, t)$ ), where $R(X, \zeta, t)$ is a Regge motivated term describing the low $X$ and $t$ behaviors, while $G_{M_{X}}^{\lambda}(X, \zeta, t)$, was obtained using a spectator model.

In order to model the $X<\zeta$ region, we observe that the higher moments of GPDs give $\zeta$-dependent constraints, in addition to the ones from the nucleon form factors. The $n=1,2,3$ moments of the NS combinations: $H^{u-d}=H^{u}-H^{d}$, and $E^{u-d}=E^{u}-E^{d}$ are available from lattice QCD $[7,8]$. They can be written in terms of the isovector components as:

$$
\begin{aligned}
H_{n}^{u-d} \equiv \int d X X^{n-1}\left(H^{u}-H^{d}\right) & =\frac{\tau\left(H_{M}^{V}\right)_{n}+\left(H_{E}^{V}\right)_{n}}{1+\tau} \\
E_{n}^{u-d} & \equiv \int d X X^{n-1}\left(E^{u}-E^{d}\right)=\frac{\left(E_{M}^{V}\right)_{n}-\left(E_{E}^{V}\right)_{n}}{1+\tau}
\end{aligned}
$$

where the l.h.s. quantities are obtained from the lattice moments calculations, whereas $\left(H_{M(E)}^{V}\right)_{n}$ and $\left(E_{M(E)}^{V}\right)_{n}$ are amenable to chiral extrapolations. We used lattice calculations 
for the unpolarized GFFs obtained by the QCDSF collaboration using two flavors of $\mathcal{O}(a)$ improved dynamical fermions for several values of $t$ in the interval $0 \lesssim t \lesssim 5 \mathrm{GeV}^{2}$, and covering a range of pion mass values, $m_{\pi} \gtrsim 500 \mathrm{MeV}^{2}$. Similarly to previous evaluations [8] the GFFs for both $H$ and $E$, display a dipole type behavior for all three $n$ values, the value of the dipole mass increasing with $n$. We performed an extrapolation by extending to the $n=2,3$ moments a simple ansatz proposed in [10] for the nucleon form factors that: i) uses the connection between the dipole mass and the nucleons radius; ii) introduces a modification of the non analytic terms in the standard chiral extrapolation that suppresses the contribution of chiral loops at large $m_{\pi}$. Despite its simplicity, the ansatz seems to reproduce both the lattice results trend at large $m_{\pi}$ while satisfying the main physical criteria $i$ ) and ii). Our results for the dipole mass at $n=2$ are shown in Fig.1.

\section{Reconstruction from Bernstein Polynomials}

Similarly to the PDFs case [11], with a finite number of moments in hand, one can use reconstruction methods attaining weighted averages of the GPDs, around average ranges of $X$. The weights are provided by the complete set of Bernstein polynomials.

In Fig.2 we show $H^{u-d}$ reconstructed using the available lattice moments. We performed the procedure in the $X<\zeta$ region only using:

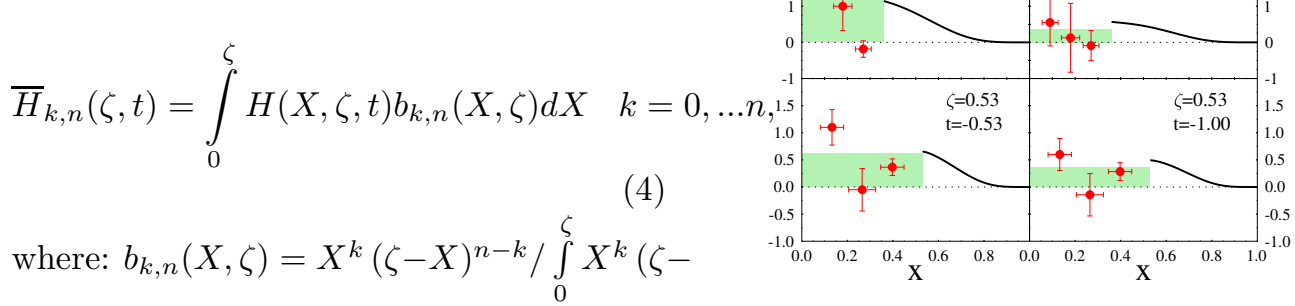

$X)^{n-k} d X$, and we used subtracted moments, defined as:

$\left(H_{n}\right)_{X<\zeta}=H_{n}-\int_{\zeta}^{1} H^{I}(X, \zeta, t) X^{n} d X$,

Figure 2: (color online) Comparison of $H^{u-d}$ for different values of $\zeta$ and $-t \equiv t_{\min }=$ $0.035,0.073,0.18,0.53 \mathrm{GeV}^{2}$ (left panel), and (5) $-t=-1 \mathrm{GeV}^{2}$ (right panel), calculated using the procedure described in the text.

where $H_{n}$ are the Mellin moments, and $H^{I}(X, \zeta, t)$ was obtained from Eq.(1). For $n=2, k=0,1,2$, the reconstruction proce- 
dure yields [12]:

$$
\begin{aligned}
& \bar{H}_{02}\left(\zeta X_{02}\right)=\frac{1}{\zeta^{3}}\left\{3 A_{10}^{\zeta} \zeta^{2}-6 A_{20}^{\zeta} \zeta+3\left[A_{30}^{\zeta}+\left(-\frac{2 \zeta}{2-\zeta}\right)^{2} A_{32}\right]\right\}, \\
& \bar{H}_{12}\left(\zeta X_{12}\right)=\frac{1}{\zeta^{3}}\left\{6 A_{20}^{\zeta} \zeta-6\left[A_{30}^{\zeta}+\left(-\frac{2 \zeta}{2-\zeta}\right)^{2} A_{32}\right]\right\}, \\
& \bar{H}_{22}\left(\zeta X_{22}\right)=\frac{1}{\zeta^{3}}\left\{3 A_{30}+\left(-\frac{2 \zeta}{2-\zeta}\right)^{2} A_{32}\right\}
\end{aligned}
$$

where $X_{01}=0.25, X_{02}=0.5, X_{03}=0.75$, and $A_{10}, A_{20}, A_{30}, A_{32}$ are the GFFs from Ref.[7].

In conclusion, we provided a fully quantitative parametrization of the NS GPDs, valid in the region of Jefferson Lab experiments [6] that, differently from model calculations, and for the first time to our knowledge, makes use of experimental data in combination with lattice results. Given the paucity of current direct experimental measurements of GPDs, our goal is to provide more stringent, model independent predictions that will be useful both for model builders, in order to understand the dynamics of GPDs, and for the planning of future hard exclusive scattering experiments.

\section{Acknowledgments}

We thank Ph. Haegler, P. Kroll and G. Schierholz for useful comments. We are also grateful to J. Zanotti for providing us with the recent lattice calculations from the QCDSF collaboration. This work is supported by the U.S. Department of Energy grant no. DEFG02-01ER41200 and NSF grant no.0426971.

\section{References}

[1] Slides: http://indico. cern. ch/contributionDisplay $\cdot$ py? contribId=90\&sessionId=8\&conf Id=9499

[2] M. Diehl, Phys. Rept. 388, 41 (2003).

[3] A. V. Belitsky and A. V. Radyushkin, Phys. Rept. 418, 1 (2005)

[4] M. Burkardt, Int. J. Mod. Phys. A 18, 173 (2003); ibid Phys. Rev. D 62, 071503 (2000) [Erratum-ibid. D 66, $119903(2002)]$.

[5] X. D. Ji, Phys. Rev. Lett. 78, 610 (1997) [arXiv:hep-ph/9603249].

[6] C. Munoz Camacho et al. [Jefferson Lab Hall A Collaboration], Phys. Rev. Lett. 97, 262002 (2006)

[7] M. Gockeler et al. [QCDSF Collaboration], Phys. Rev. D 73, 014513 (2006), and references therein.

[8] R. G. Edwards et al. [LHPC Collaboration], PoS LAT2005, 056 (2006) [arXiv:hep-lat/0509185], and references therein.

[9] S. Ahmad, H. Honkanen, S. Liuti and S.K. Taneja, Phys. Rev. D 75, 094003 (2007)

[10] J. D. Ashley, D. B. Leinweber, A. W. Thomas and R. D. Young, Eur. Phys. J. A 19, 9 (2004).

[11] F.J. Yndurain, Phys. Lett. 74B (1977) 68.

[12] S. Ahmad, H. Honkanen, S. Liuti and S.K. Taneja, in preparation. 